Danmer Paulino Maza Quinones

\title{
Processo de revestimento de cilindros
}

fotossensíveis

Tese apresentada ao Programa de Pós-graduação em Engenharia Mecânica do Departamento de Engenharia Mecânica da PUCRio como requisito parcial para obtenção do título de Doutor em Engenharia Mecânica

Orientador: Prof. Márcio da Silveira Carvalho 


\section{Danmer Paulino Maza Quinones}

\section{Processo de revestimento de cilindros fotossensíveis}

Tese apresentada ao Programa de Pós-graduação em Engenharia Mecânica do Departamento de Engenharia Mecânica do Centro Técnico Científico da PUC-Rio como requisito parcial para obtenção do título de Doutor em Engenharia Mecânica. Aprovada pela Comissão Examinadora abaixo assinada.

Prof. Márcio da Silveira Carvalho Orientador Departamento de Engenharia Mecânica - PUC-Rio

Prof. Angela Ourivio Nieckele Departamento de Engenharia Mecânica - PUC-Rio

Prof. Mônica Feijó Naccache Departamento de Engenharia Mecânica - PUC-Rio

Prof. Francisco Ricardo da Cunha Departamento de Engenharia Mecânica - UNB

Prof. Márcio Arab Murad Departamento de Matemática Aplicada - LNCC

Prof. José Eugênio Leal Coordenador Setorial do Centro Técnico Científico - PUC-Rio 
Todos os direitos reservados. É proibida a reprodução total ou parcial do trabalho sem autorização da universidade, do autor e do orientador.

\section{Danmer Paulino Maza Quinones}

Graduou-se em Engenharia Mecânica-Elétrica na Universidad Nacional de Ingeniería- UNI (Lima, Perú) em 2001. Concluiu o Mestrado na PUC-Rio (Rio de Janeiro, Brasil) em 2005 na área de Termociências estudando a Elastohidrodinâmica dos processos de revestimento por extrusão, utilizando o Método de Elementos Finitos.

Ficha Catalográfica

Maza Quinones, Danmer Paulino

Processo de revestimento de cilindros fotossensíveis / Danmer Paulino Maza Quinones; orientador: Márcio da Silveira Carvalho. - Rio de Janeiro : PUC-Rio, Departamento de Engenharia Mecânica, 2009.

v., 130 f: il. (color) ; $30 \mathrm{~cm}$

Tese (doutorado) - Pontifícia Universidade Católica do Rio de Janeiro, Departamento de Engenharia Mecânica.

Inclui referências bibliográficas.

1. Engenharia Mecânica - Tese. 2. Processo de revestimento;. 3. filmes finos;. 4. problemas de superfície livre;. 5. método de diferenças finitas.. I. Carvalho, Márcio da Silveira. II. Pontifícia Universidade Católica do Rio de Janeiro. Departamento de Engenharia Mecânica. III. Título. 


\section{Agradecimentos}

Ao meu orientador, professor Márcio Carvalho, pelo grande apoio e confiança depositada.

Ao já falecido Professor L. E. Scriven e ao professor Satish Kumar pela oportunidade concedida para realizar os testes experimentais no Laboratório de Revestimento da Universidade de Minnesota. A Jackie Pesch e Wieslaw Suszinsky pelo apoio ao desenvolvimento das experiências. Agradeço também aos amigos Jaewook, Kris, Benson, Hiroaki, Hiroshi e Joshifumi pela amizade durante minha permanência em Minneapolis.

Agradeço também aos professores membros da banca, pela participação, comentários e sugestões feitas ao trabalho apresentado.

Aos professores e funcionários do Departamento de Engenharia Mecânica da PUC-Rio pelos ensinamentos e pela ajuda ao longo destes quatro anos.

À todos os meus irmãos e a toda minha família no Perú, pelo incentivo, estima e carinho sempre manifestados. Minha eterna gratidão à minha querida esposa Satya Devidasi, pelo carinho e incessante apoio e também para toda a família Ribeiro, Mazza e Pernambuco.

À todos os meus amigos, conhecidos em diversas circunstâncias durante minha permanência no curso de doutorado, eles fizeram certamente do caminho percorrido muito mais agradável: Melisa, Joel, Sigyfredo, Teresa, Juliana, Marlon, Epifanio, Mao, Raúl, Júlio, Flávio, André, Jane, Aline, Priscilla, Eduardo, Ranena, Fred e os demais alunos dos professores Márcio, Paulo e Mônica (Grupo GREO).

Finalmente minha gratidão ao CNPq e à PUC-Rio, pelos auxílios concedidos, sem os quais este trabalho não poderia ter sido realizado. 


\section{Resumo}

Maza Quinones, Danmer Paulino; Carvalho, Márcio da Silveira. Processo de revestimento de cilindros fotossensíveis. Rio de Janeiro, 2009. 130p. Tese de Doutorado - Departamento de Engenharia Mecânica, Pontifícia Universidade Católica do Rio de Janeiro.

Cilindros fotossensíveis são usados nos processos de impressão e particularmente na impressão eletrofotográfica. O revestimento é aplicado ao cilindro em forma líquida antes de solidificar-se. O líquido é aplicado ao cilindro através de um aplicador de agulha que se translada ao longo da direção axial do cilindro. Durante este processo o cilindro gira em torno de seu próprio eixo levando a uma deposição da tira de líquido sobre a superfície do cilindro, em padrão espiral. Para ajudar a distribuir o líquido lateralmente e assim melhorar a uniformidade da espessura, cada tira de líquido aplicada pela agulha passa por meio de uma lâmina flexível. Este processo leva a um revestimento que apresenta um padrão espiral na espessura da camada revestida que pode causar defeitos no processo eletrofotográfico.O conhecimento, de forma fundamental, do escoamento é vital para a otimização do processo.

Um modelo teórico de escoamento de filmes finos sobre superfícies cilíndricas em rotação com uma porta de injeção de líquido em movimento é apresentado. Este modelo é baseado na teoria de lubrificação considerando um filme precursor na frente da linha de contato aparente. A espessura de filme revestido foi obtida através da solução de uma equação diferencial parcial não linear de quarta ordem usando o método de diferenças finitas de segunda ordem. A discretização do tempo foi feita pelo método implícito de CrankNicholson. A discretização do sistema a cada passo do tempo leva a um sistema de equações algébricas não lineares que foi resolvido pelo método de Newton. Os resultados mostram como a uniformidade da camada depositada varia com os parâmetros do processo e com as propriedades do líquido.

\section{Palavras-chave}

Processo de revestimento; filmes finos; problemas de superfície livre; método de diferenças finitas. 


\section{Abstract}

Maza Quinones, Danmer Paulino; Carvalho, Márcio da Silveira (Advisor). Coating process of photosensitive cylinders. Rio de Janeiro, 2009. 130p. DSc. Thesis — Departamento de Engenharia Mecânica, Pontifícia Universidade Católica do Rio de Janeiro.

Photosensitive cylinders are used in printing arts and more particularly in electrophotographic printing (e.g. xerographic copy). The photosensitive coating is applied to the cylinder in liquid form, before it is solidified. The liquid is applied to the cylinder through a needle applicator that translates along the direction of the cylinder axis. The cylinder rotates during this process in order to cover the entire surface. Therefore, the liquid is applied in a spiral pattern. In order to help spreading of the liquid over the cylinder surface and to improve the thickness uniformity, each liquid stream applied from the needle passes under a flexible blade. This process leads to a coating that presents a spiral pattern on the deposited layer thickness, which can cause defects on the electrophotographic process. The complete understanding of the flow is vital to the optimization of the process.

A theoretical model of the thin film flow over the surface of a rotating cylinder is presented here. It is based on the lubrication approximation considering a thin precursor film in front of the apparent contact line. The resulting non-linear fourth-order PDE for the film thickness was solved by a second-order finite difference method. The time discretization was done by the implicit Crank-Nicholson scheme. The non-linear algebraic equation at each time step was solved by Newton's method. The results show how the uniformity of the deposited layer varies with process parameters and liquid properties.

\section{Keywords}

Coating process; thin film; free boundary problem; finite difference method. 


\section{Sumário}

$\begin{array}{ll}\text { Sumário das notações } & 15\end{array}$

$1 \quad$ Introdução $\quad 18$

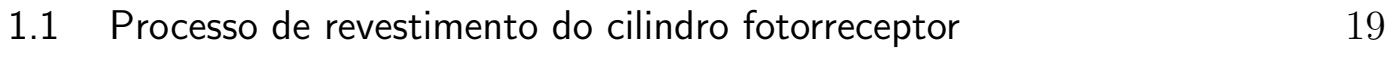

1.1.1 Descrição dos sistemas de eletrofotografia ou xerografia 19

$\begin{array}{ll}1.1 .2 & \text { Cilindro fotorreceptor }\end{array}$

1.1.3 Características físicas do cilindro fotorreceptor OPC 21

1.1.4 Vantagens da tecnologia de fotocondutores orgânicos 23

1.1.5 Processo de revestimento do cilindro fotorreceptor 24

1.2 Escopo e Roteiro da Tese 26

2 Formulação matemática e modelagem computacional do escoamento de filme fino sobre uma placa plana $\quad 29$

2.1 Formulação matemática 29

2.2 Equações adimensionalizadas 33

2.3 Descrição da linha de contato dinâmica 34

2.4 Modelagem computacional 36

2.4.1 Discretização espacial 37

2.4.2 Termo de tensão superficial 38

2.4.3 Discretização dos termos gravitacionais 40

2.4.4 Discretização do tempo 40

2.4.5 Condição de contorno 42

2.4.6 Método da solução 43

2.4.7 Critérios de convergência 44

2.5 Resultados com uma porta de injeção 45

2.5.1 Teste da malha 46

2.5.2 Efeito da espessura do filme precursor 48

2.5.3 Validação 48

2.5.4 Efeito do parâmetro alpha 50

2.5.5 Efeito da vazão de injeção 51

2.5.6 Efeito do Bo 54

3 Análise experimental do escoamento sobre uma placa plana com duas portas de injeção: interação entre gotas $\quad 57$

$\begin{array}{lll}3.1 & \text { Dispositivo experimental } & 57\end{array}$

3.2 Preparação das soluções 59

$\begin{array}{lll}3.3 & \text { Resultados } & 61\end{array}$

3.3.1 Resultado com injeção de glicerina pura 62

3.3.2 Resultado com injeção de solução com $97 \%$ de glicerina 64

3.3.3 Resultado com injeção de solução com 80\% de glicerina 66

4 Formulação matemática e modelagem computacional do escoamento de filmes finos em cilindros com rotação $\quad \mathbf{6 9}$

$\begin{array}{lll}4.1 & \text { Cenário } & 69\end{array}$ 
$\begin{array}{lll}4.2 & \text { Formulação } & 74\end{array}$

4.3 Teoria de lubrificação tri-dimensional 76

$\begin{array}{lll}4.4 & \text { Solução numérica } & 79\end{array}$

4.4.1 Discretização espacial $\quad 80$

4.4.2 Discretização dos termos da equação de evolução 80

4.4.3 Discretização do tempo 81

$\begin{array}{ll}\text { 4.4.4 Condição de contorno } & 82\end{array}$

4.5 Validação $\quad 82$

4.5.1 Teoria de Moffat 83

4.5.2 Instabilidade Rayleight-Taylor 86

$\begin{array}{lll}4.6 & \text { Resultados } & 90\end{array}$

4.6.1 Efeito de advecção do líquido por rotação do cilindro 90

4.6.2 Efeito de espalhamento na superfície do cilindro 92

4.6.3 Efeito da relação das velocidades $V_{i n j}$ e $W$

$\begin{array}{ll}\text { 4.6.4 Efeito de nivelamento } & 95\end{array}$

5 Formulação matemática e modelagem computacional de escoamento $\begin{array}{ll}\text { com pseudo-solidifição } & 101\end{array}$

$\begin{array}{lll}5.1 & \text { Formulação } & 103\end{array}$

5.2 Teoria de lubrificação bi-dimensional 106

5.3 Equação da evolução da espessura do líquido injetado com o tempo 112

5.4 Solução numérica 114

5.4.1 Mapeamento temporal e espacial de $\eta \quad 116$

$\begin{array}{lll}5.5 \text { Resultados } & 116\end{array}$

$\begin{array}{lll}\text { 5.5.1 Efeito de nivelamento } & 118\end{array}$

6 Comentários finais e sugestões $\quad 122$

6.1 Comentários finais 122

$\begin{array}{lll}6.2 & \text { Sugestões para trabalhos futuros } & 123\end{array}$

$\begin{array}{ll}\text { Referências Bibliográficas } & 125\end{array}$ 


\section{Lista de figuras}

1.1 Desenho de um típico sistema de impressão eletrográfico, vista lateral (a) e uma sequência aproximada dos passos utilizados por Carlson no processo eletrográfico, vista mista lateral e do topo (b)

1.2 Estrutura genérica de um típico fotocondutor orgânico (OPC) e a descrição das diferentes camadas. Este tipo de desenho permite flexibilidade na escolha de materiais de maneira a otimizar a performance do dispositivo

1.3 Vista simplificada dos componentes de um sistema que reveste cilindros fotorreceptores; (retirada da U.S. Pat. No. 6,521,330)[1]

1.4 Fotografias do processo de revestimento de cilindros fotorreceptores (fornecidas pela Xerox Corporation) e a descrição de alguns elementos deste processo; (a) no tempo inicial do processo; (b) num tempo intermediário.

1.5 Superfície ondulada da camada revestida.

2.1 Esquema do escoamento 3D de filme fino, num plano inclinado, gerado por duas portas de injeção.

2.3 Esboço do perfil de um líquido perto da LCD e a distribuição parabólica do vetor de velocidade $u_{x}(z)$ para (a) método do filme precursor e (b) método de relaxação. Nota-se que em (b) a velocidade $u_{x}(z)$ não é zero na superfície (deslizamento).

2.4 Malha típica mostrando o estêncil formado por 13 pontos em torno do nó $k$ que serão utilizados para avaliar as derivadas de maior ordem. 37

2.5 Presença de altos gradientes de $h$ perto da LCD pelo uso do modelo de filme precursor.

2.6 Esquema que identifica a localização dos coeficientes difusivos. $\quad 39$

2.7 Avaliação da condição de contorno com aplicação de nós fictícios.

2.8 O escoamento de filme fino por injeção em superfície plana com os principais parâmetros adimensionais

2.9 Teste de malha com $B o=172,05 ; C a=0,33 ; H_{f}=1,67 \times 10^{-3}$; $\Phi=1,8 ; R_{f}=1,67 \times 10^{-2}$ e considerando o ângulo $\alpha=15^{\circ}$. Critério de passo de tempo: a) $D t$ variável.

2.10 Teste de malha com $B o=172,05 ; C a=0,33 ; H_{f}=1,67 \times 10^{-3}$; $\Phi=1,8 ; R_{f}=1,67 \times 10^{-2}$ e considerando o ângulo $\alpha=15^{\circ}$. Critério de passo de tempo: b) $D t$ constante e variável.

2.11 Teste do parâmetro $H_{f}$ com $B o=172,05 ; C a=0,33 ; \Phi=1,8$; $R_{f}=1,67 \times 10^{-2}$ e considerando o ângulo $\alpha=15^{\circ}$.

2.12 Resultado numérico mostrando o efeito do $\alpha$ considerando os seguintes parâmetros $\Phi=3,45 ; B o=174,24 ; C a=0,33$; $H_{f}=1,67 \times 10^{-3} ; R_{f}=1,67 \times 10^{-2}$

2.13 Comparação de resultados em função da vazão de injeção para um fluido com $B o=174,24 ; C a=0,33 ; H_{f}=1,67 \times 10^{-3}$; $R_{f}=1,67 \times 10^{-2}$ para diferentes $\alpha=15^{\circ} ; 30^{\circ} ; 60^{\circ} ; 75^{\circ}$ e $90^{\circ}$. (a) 3,45 , (b) 6,9 , (c) 13,8 ; (d) 20,7 
2.14 Gráfico da velocidade media em função do parâmetro alpha para um fluido com $B o=174,24 ; C a=0,33 ; H_{f}=1,67 \times 10^{-3}$; $R_{f}=1,67 \times 10^{-2}$.

2.15 Gráfico de $X_{f}$ e $Y_{\max }$ para 3 tipos de fluidos mantendo os mesmos parâmetros $H_{f}=1,67 \times 10^{-3} ; R_{f}=1,67 \times 10^{-2} ; \Phi=2,2 \mathrm{e}$ $\alpha=15^{\circ}$.

2.16 Perfis de espessura nas direções $X_{f}$ e $Y_{\max }$ para 4 diferentes números de $B o$, no instante em que atingem $X_{f}=0,234$ mantendo constantes os outros parâmetros $H_{f}=1,67 \times 10^{-3} ; R_{f}=$ $1,67 \times 10^{-2} ; \Phi=2,2$ e $\alpha=15^{\circ}$.

3.1 Fotografia do dispositivo experimental utilizado. 58

3.2 Esquema ilustrativo da bancada experimental.

3.3 Sequência de imagens mostrando a interação de duas gotas numa placa de acrílico inclinada a $\alpha=15^{\circ}$. a) injeção não sincronizada. b) injeção bem sincronizada, onde a coalescência simétrica é observada. 60

3.4 Imagem da área de injeção de líquido numa placa inclinada, onde as portas de injeção são visíveis; $\alpha=15^{\circ} ; \Gamma=0,5 \mathrm{ml} / \mathrm{min}$; porta de injeção $2 R_{f}=1,0 \mathrm{~mm}$

3.5 Visualização do topo do experimento e a comparação com os resultados obtidos numericamente: caso glicerina $100 \%$; $\alpha=15^{\circ}$; $\Gamma=0,5 \mathrm{ml} / \mathrm{min}$. a) final do primeiro estágio e b) durante o segundo estágio.

3.6 Visualização do topo do experimento e a comparação com os resultados obtidos numericamente: caso glicerina $100 \%$; $\alpha=15^{\circ}$; $\Gamma=0,5 \mathrm{ml} / \mathrm{min}$. c) durante o terceiro estágio.

3.7 Comparação dos resultados numéricos e experimentais da evolução da frente de avanço $X_{f}$, com alpha $=15^{\circ}$ e $\Gamma=0,5 \mathrm{ml} / \mathrm{min}$.

3.8 Frente de avanço $X_{f}$ para diferentes taxas de injeção volumétrica: Caso Glicerina pura; $\alpha=15^{\circ}$.

3.9 Gráfico comparativo, da evolução da frente de avanço, da injeção de líquido: desde duas portas de injeção com $\Gamma=0,5 \mathrm{ml} / \mathrm{min}$ e uma porta de injeção com uma porta de injeção com $\Gamma=1,0 \mathrm{ml} / \mathrm{min}$, ambas com $\alpha=15^{\circ}$.

3.10 Comparação numérica e experimental da evolução da frente de avanço $X_{f}$ para diferentes taxas de injeção volumétrica: Caso Glicerina 97\%; alpha $=15^{\circ}$.

3.11 Comparação numérica e experimental da evolução da frente de avanço $X_{f}$ para diferentes taxas de injeção volumétrica:Caso Glicerina $80 \% ; \alpha=15^{\circ}$.

3.12 Gráfico comparativo, da evolução da frente de avanço, de líquidos com diferentes viscosidades com $\alpha=15^{\circ}$ e $\Gamma=1,0 \mathrm{ml} / \mathrm{min}$.

4.1 Esquema do sistema de coordenada fixa do cilindro, mostrando a direção da rotação do cilindro e a força gravitacional.

4.2 Escoamento laminar numa região de solução descontínua mostrada por Dieber \& Cerro [2]. No desenho a espessura do filme não está em escala. 
4.3 Formações tipo dentes de tubarão (shark teeth) ao longo do eixo axial do cilindro. Para um fluido com $\mu=49 \mathrm{cP}, \Omega=3,2 \mathrm{c} / \mathrm{s}$ e uma relação de volume de líquido com volume total do cilindro $\eta V=6,0 \%$. Retirado do trabalho experimental de Thoroddsen \& Mahadevan [3].

4.4 Formação de paredes ou cortinas radiais no interior de um cilindro. Para um fluido com a) $\mu=120 \mathrm{cP}, \Omega=4,9 \mathrm{c} / \mathrm{s}$ e uma relação de volume de líquido com o volume total do cilindro $\eta_{V}=18,2$ e b) $\mu=28 \mathrm{cP}, \Omega=7,2 \mathrm{c} / \mathrm{s}$ e uma relação de volume de líquido com o volume total do cilindro $\eta_{V}=32,7 \%$. Retirados do trabalho experimental de Thoroddsen \& Mahadevan [3].

4.5 Gráfico do sistema de revestimento que mostra o cilindro giratório num sistema de coordenada fixa sem rotação.

4.6 Representação da porta de injeção no interior de um cilindro.

4.7 Efeito da rotação do cilindro. O líquido que está sendo formado na base por efeito gravitacional é carregado na direção da rotação. Para cada tipo de velocidade $W$ é obtido um perfil diferente de líquido, se a velocidade for baixa forma-se uma maior acumulação de líquido na base do cilíndro, se a velocidade for aumentada, a acumulação será menor.

4.8 Resultados dos valores da espessura máxima e mínima do filme revestido e também da posição $\theta$ em função da taxa de rotação $W$. (a) Máxima $(*)$ e mínima $(+)$ espessura atingida. (b) Localização das espessuras máxima $(*)$ e mínima $(+)$ espessura.

4.9 Configuração instável por efeito gravitacional de uma camada de filme embaixo de uma superfície horizontal (Ex: teto de um quarto molhado). a) camada de líquido no instante $t=0$. b) nova configuração do líquido em $t=t^{*}$

Resultados experimentais e teóricos do comprimento de onda em diferentes raios de cilindros, ambos em forma adimensional, dados por Bruyn [4].

4.11 Vista do perfil da malha na base do cilindro estacionário mostrando a formação de gotas com comprimento de onda $\lambda=10,2$.

4.12 Teste do efeito de advecção do líquido pela rotação do cilindro com $W=1,5$. a) Vista de perfil mostrando as posições das portas de injeção testadas, b) Domínio $\theta$ vs $y$ da malha estendida mostrando a injeção de líquido na posição $\left(\theta_{c p}, y_{c p}\right)$ e c) Perfil do filme gerado pela injeção contínua e advectada pela rotação do cilindro para as diferentes posições indicadas em a).

4.13 Comparação dos perfis da tira revestida para $W=3,0$ e $\Gamma=0,005$ para as diferentes posições da porta de injeção dada na Fig. 4.12a). 92

4.14 Efeito de espalhamento numa tira de líquido sobre a superfície do cilindro. a) corte transversal da tira líquida. b) vista de topo da tira revestida.

4.15 Efeito da velocidade da porta de injeção, $V_{i n j}$ : a) $V_{i n j}=40 \times 10^{-3}$; b) $V_{i n j}=4,0 \times 10^{-3}$ e c) $V_{i n j}=0,4 \times 10^{-3}$, representadas em curvas de nível e em forma tri-dimensional. 
4.16 Espessura de filme revestido no domínio $\theta-y$ após uma escolha apropriada nos valores dos parâmetros $V_{i n j}=0,001, \Gamma=0,001 \mathrm{e}$ $W=3,0$ para o caso de $R_{f}=0,25$.

4.17 Gráfico do perfil da espessura do filme depositado em $\theta=\pi / 2$ (Corte (A-A)): a) no instante no qual a porta de injeção atinge a distância final axial. b) No seguinte intervalo de tempo $t$.

4.18 a) Espessura média ao longo do tempo, medida em $\theta=\pi / 2$ e $\theta=3 \pi / 2$ para $B o=1,0$. b) Tempo $T_{\text {crit }}$ para diferentes valores de $B o$.

98

4.19 Perfil da espessura do filme em $T_{\text {crit }}$ para $B o=1,0$.

4.20 Perfil do filme revestido quando $\mathrm{W}=1,0 ; V_{i n j}=0.001 / 3 \mathrm{e}$ $\Gamma=0,0035$

4.21 Comparação de perfil do filme revestido para diferentes taxas de rotação para $B o=1,0$.

4.22 Espessura média ao longo do tempo, medida em $\theta=\pi / 2$ e $\theta=3 \pi / 2$ para $B o=1,0$.

5.1 a) Viscosidade em função da concentração de massa do solvente.

b) Variação da fração de massa de um sistema com solvente volátil (tolueno) obtida por Drike \& Wang [5].

5.2 Viscosidade como uma função do tempo. $\eta_{\text {Ini }}=1,0$ e $\eta_{\text {Fin }}=1000$ para um $t_{\eta_{\text {Ini }}}=120$ e $t_{\eta_{\text {Fin }}}=1500$.

5.3 Mapeamento de $\eta$.

5.4 Gráfico do perfil da espessura do filme depositado em $\theta=\pi / 2$ (Corte (A-A)): a) no instante no qual a porta de injeção atinge a distância final axial. b) No seguinte intervalo de tempo $t$ com o cilindro estacionário.

5.5 Perfis de espessura obtidos para os casos $B o=1,0$ e $B o=0,01$ para $t=1,6 \times 10^{3}$. Comparação do caso base sem considerar solidificação e os casos obtidos neste capítulo, para os mesmos parâmetros de operação.

5.6 Comparação dos perfis de espessura obtidos em $t=1,45 \times 10^{4}$ medida em $\theta=\pi / 2$. Com pseudo-solidificação representada com linha tracejada e sem pseudo-solidificação com linha pontilhada.

5.7 Comparação da espessura média ao longo do tempo, medida em $\theta=\pi / 2$ e $\theta=3 \pi / 2$ para $B o=1,0$, obtidas pela analise do capítulo 4 e 5 .

5.8 Espessura média ao longo do tempo, medida em $\theta=\pi / 2$ e $\theta=3 \pi / 2$ para $B o=1,0$. Com $W=1,0$ (superior) e com $W=3,0$ (inferior). 


\section{Lista de tabelas}

2.1 Casos utilizados para o teste da malha 48

2.2 Comparação do expoente power law, $m$, entre resultados analíticos e numéricos do espalhamento de uma gota de líquido (volume constante):

2.3 Comparação do expoente power law, $m$, entre resultados analíticos e numéricos considerando injeção contínua de líquido:

2.4 Valores típicos utilizados para avaliar o efeito do número de Bond, Bo

3.1 Propriedades dos fluidos

5.1 Valores dos parâmetros utilizados. 
A Computação Científica é a arte de utilizar a intuição física, os algoritmos e teoremas matemáticos e a moderna tecnologia dos computadores para construir e explorar modelos realísticos dos problemas que surgem na Ciência e na Engenharia

Garret Birkhoff, Algebra linear computacional. 


\section{Sumário das notações}

\section{Símbolos Romanos}

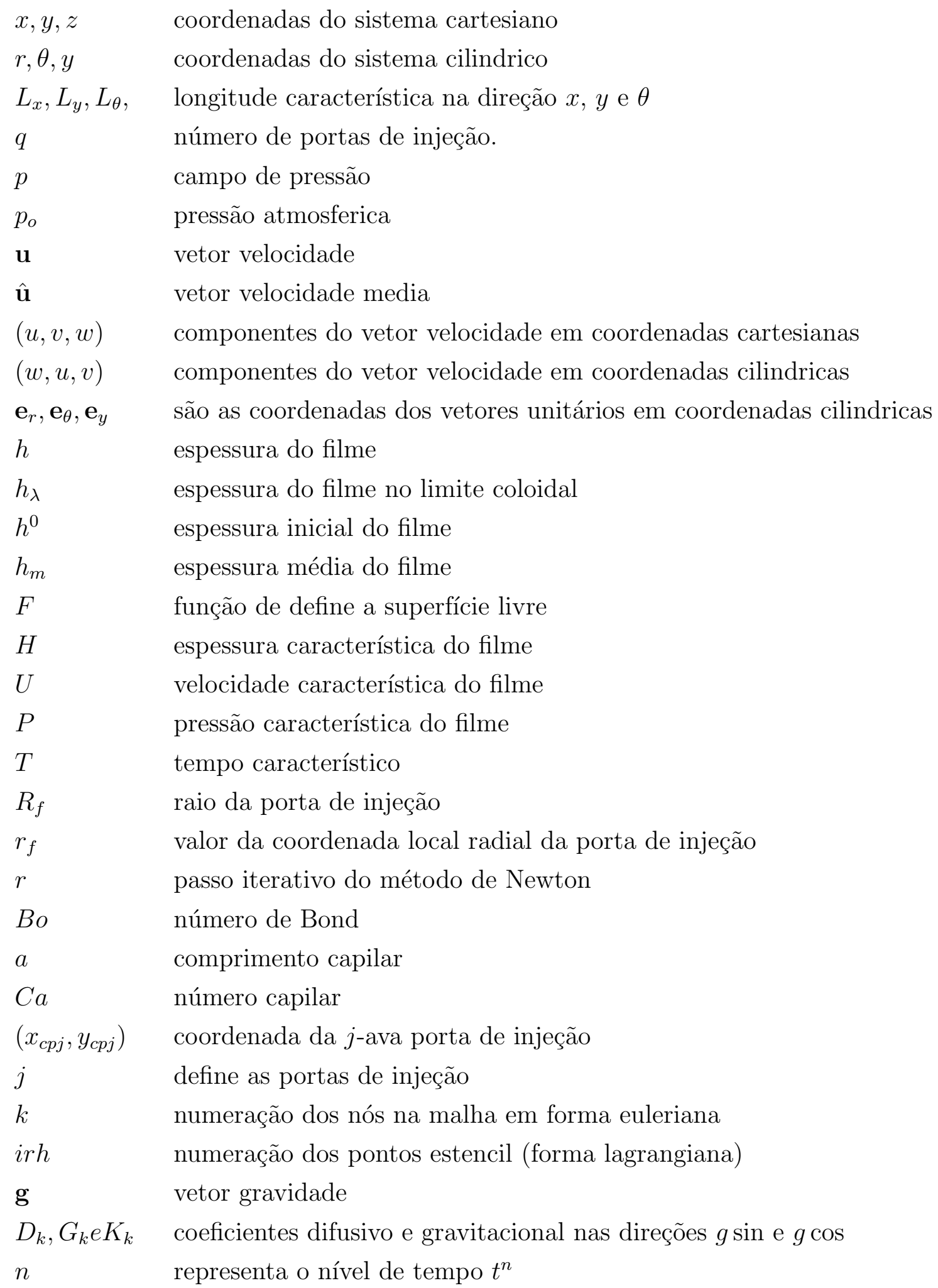




\begin{tabular}{|c|c|c|}
\hline & $\mathbf{R}$ & vetor de residuos \\
\hline & b & vetor dos parâmetros \\
\hline & $\mathbf{J}$ & matriz jacobiana \\
\hline & $Y_{\max }$ & distancia transversal maxima \\
\hline & $X_{f}$ & posição da frente de avance \\
\hline & $H_{f}$ & espessura do filme precursor \\
\hline & $V_{f}$ & velocidade media da frente de avance \\
\hline & $Q$ & vazão \\
\hline & $R$ & raio do cilindro \\
\hline & $r$ & posição radial arbitraria \\
\hline & $V_{\text {need }}$ & velocidade de translação da porta de injeção \\
\hline & $W$ & taxa de rotação adimensional \\
\hline & $W_{c}$ & taxa de rotação critica adimensional \\
\hline & $M$ & viscosidade adimensional \\
\hline & $k$ & número de onda na direção $x$ \\
\hline & $y_{c p}$ & posição axial da porta de injeção \\
\hline & $N x, N \theta, N y$ & numero de elementos na direção correspondente \\
\hline & $x_{A}$ & fração em massa do solvente \\
\hline & $\hat{\mathbf{n}}$ & vetor normal à superficie \\
\hline & $\hat{\mathbf{t}}$ & vetor tangencial à superficie \\
\hline & $\mathrm{D}$ & tensor taxa de deformação \\
\hline & $\mathfrak{R}$ & número de Reynolds \\
\hline & $\mathcal{O}$ & ordem de grandeza \\
\hline & $U_{\Omega}$ & velocidade relativa entre o substrato e o liquido \\
\hline
\end{tabular}

\section{Símbolos Gregos}

$\begin{array}{ll}\rho & \text { densidade } \\ \mu, \nu & \text { viscosidade dinâmica e cinemática } \\ \sigma & \text { tensão superficial } \\ \epsilon & \text { relação de aspecto para aplicar a teoria de lubrificação, H/L ou H/R } \\ \Phi & \text { função velocidade de injeção } \\ \Gamma & \text { vazão de injeção volumetrica } \\ \alpha & \text { ângulo de inclinação do plano } \\ \theta_{L C D} & \text { ângulo de contato dinâmico } \\ \theta_{C N} & \text { esquema de integração } \theta \text { ou de Crank-Nicholson } \\ \tau & \text { tensor das tensões } \\ \Omega & \text { taxa de rotação }\end{array}$


$\Omega_{c} \quad$ taxa de rotação crítica

$\lambda \quad$ comprimento de onda

$\beta \quad$ amplitude de perturbação

$\omega \quad$ taxa de crescimento da instabilidade

$\psi \quad$ ângulo de direção de aporte de líquido

$\eta V \quad$ relação de volume de líquido com o volume total do cilindro

$\theta_{c p} \quad$ posição azimutal da porta de injeção

$\Delta x, \Delta \theta, \Delta y$ tamanhos dos elementos na direção correspondente

$\eta_{o}, \eta_{i n i} \quad$ viscosidade de solidificação inicial

$\eta_{s}, \eta_{\text {fin }} \quad$ viscosidade de solidificação final

$\kappa$

curvatura media da superfície livre

$\varepsilon \quad$ erro relativo para um passo do tempo $\Delta t^{n}$

$\xi \quad$ tolerância no critério de convergencia 